\title{
Modeling Redundancy-based Routing in Delay Tolerant Networks
}

\author{
Yong $\mathrm{Liao}^{\dagger}$, Kun Tan*, Zhensheng Zhang ${ }^{\ddagger}$, Lixin $\mathrm{Gao}^{\dagger}$ \\ ${ }^{\dagger}$ Dep. of Electrical \& Computer Eng. \\ University of Massachusetts \\ Amherst, MA 01003, USA \\ email: \{yliao,lgao\}@ecs.umass.edu \\ ${ }^{*}$ Microsoft Research Asia \\ Sigma Center, No. 49 Zhichun Rd \\ Beijing 100080, China \\ email: kuntan@microsoft.com \\ ${ }^{\ddagger}$ San Diego Research Center \\ Suite A, 6696 Mesa Ridge Rd \\ San Diego, CA 92121, USA \\ email: zzhang@ieee.org
}

\begin{abstract}
Delay Tolerant Networks (DTNs) are usually intermittently connected mobile wireless networks. Routing in delay tolerant networks is challenging because many well-known assumptions of traditional networks do not hold in DTNs. Various routing schemes have been proposed in literature which exploit redundancy to improve the message delivery. However, there lacks a systematical analytic work on modeling the redundancybased routing schemes for delay tolerant networks. This motivates us to develop a unified model to analyze the various redundancy-based routing schemes. In this paper, we model the message delivering process in DTNs as a continuous time Markov random process with absorbing state. With this model, we have derived the probability distribution functions of the message delivery delay for different routing schemes, including direct transmission routing, source forward, and binary forward schemes. We conduct simulations to validate our model and our analytic results. The simulations show that our model can precisely capture the characteristics of those redundancy-based routing schemes.
\end{abstract}

\section{INTRODUCTION}

Delay Tolerant Networks (DTNs) are intermittently connected mobile wireless networks in which the connectivity between nodes changes frequently due to nodes movement. Examples of this sort of network include inter-planetary networks [1], wildlife tracking and habitat monitoring sensor networks [2], and etc. In general, the DTN routing works in an extended store-and-forward manner. Messages are sent between two nodes when they encounter and wireless communication is established. Nodes may cache the messages for considerably long time before getting the chance to send them to the next hop nodes.

Because the exact contact information between any two nodes in DTN is usually unknown in advance, making right routing decisions is challenging. There is a class of routing algorithms in DTN that exploits redundancy to reduce the delivery delay and increase the successful delivery rate. One simple and straightforward redundancy-based scheme is flooding-based epidemic routing. Flooding-based routing schemes can cause too much overhead for the network and overflow the buffer of the nodes. In order to control the overhead of flooding-based routing schemes, the authors of [3] propose to dispatch a certain number of identical message copies to a fixed number of relay nodes, instead of sending to every node in the network. When a message is generated by a source node, a "quota" is attached to that message, which represents how many identical copies of the message can be inserted in the network. When a node carrying the message forwards a copy to another node, the remaining quota of the message is distributed between the two copies on the two different nodes. The node will not forward the message to somebody else if the carried message does not have enough quota.

To the best of our knowledge, there is little systematic analytic work on modeling and analyzing redundancy-based routing, although lots of routing schemes have been proposed. In this paper, we propose a model using continuous time Markov chain with absorbing state to study the performance of redundancy-based routing in delay tolerant networks. The non-absorbing states of the Markov chain is the number of relays carrying copies of the same message. When the message is successfully delivered to its destination node, the Markov chain enters its absorbing state. The transfer rate between different states depends on the message forwarding schemes and we will discuss details of the state transfer rate for different message forwarding schemes in Section IV. Our model can be used to analyze a wide range of redundancybased routing schemes. By our model, we can derive the probability distribution of the message delivery delay, which can provide rich information in design of more sophisticated redundancy-based routing schemes. Simulation results have shown that our model precisely catch the characteristics of general redundancy-based routing schemes.

The rest of this paper is organized as follows. Section II is a brief overview of the previous works related to this paper. Section III presents our model in detail. We use the Markov chain model to analyze different redundancy-based routing schemes in Section IV. The simulation results are presented in Section V. We conclude this paper in Section VI.

\section{RELATED WORK}

\section{A. Deterministic Routing}

Delay tolerant network has attracted significant attention recently. There have been many research activities in the area of DTN routing, which is a fundamental issue in DTNs [4]. The knowledge-based routing [5] formulates the DTN routing problem as moving messages across a graph with time-varying connectivity whose dynamics is predictable. Merugu et al assume the network profile can be accurately predicted over 
certain period of time and propose the space-time routing scheme in [6]. The dynamic of the networks is modeled as space-time graph. Finding the best route for message is done by looking ahead of time. Both the work in [5] and [6] are deterministic routing schemes.

\section{B. Opportunistic Routing}

The direct transmission routing scheme [7] is the simplest and most resource efficient one, in which the source node holds the message until it meets the destination node. This scheme, although has minimal overhead, may incur very long delays for message delivery. The utility based routing schemes [8] [9] [10] define parameters to represent the capability of a relay node to deliver the message and use that information to assist making the decision of forwarding message.

Single message copy opportunistic routing may have too long delivery delay. To shorten the delay, message redundancy-based routing is introduced. The flooding routing is proposed in [11] which forwards the message to all nodes in the networks. Flooding routing has the highest probability of delivery (or least latency) and the most overhead in terms of bandwidth and energy. To bound the overhead of delivering a message, Spyropoulos et al propose to spray only a fixed number of message copies into the network [3] [12]. Wang et al [13] propose to erasure code the messages into large number of blocks to achieve more diversity in forwarding. The scheme proposed in [14] integrates erasure coding into utility based routing. The schemes proposed in [13] and [14] have fixed overhead as well.

To the best of our knowledge, there lacks a systematic analytical work on modeling the redundancy-based routing, although a number of routing schemes have been proposed. The branching process is used to model the epidemic routing in DTNs [15]. In [12] the authors give upper and lower bound of the message delivery delay in multi-copy message routing scheme. Our work is different from the previous work in that we propose a model which can be used in modeling a wide range of redundancy-based routing scheme. By this model, we can derive the message delivery delay distribution, which can be a rich source of information for improving the performance of redundancy-based routing schemes.

\section{Modeling Redundancy-BASED Routing}

\section{A. Assumptions and Notations}

In order to simplify the analysis, we have a set of assumptions here. We assume nodes move according to the random waypoint mobility model. The storage capacity of each node is large enough and no cache replacement occurs. For any node, the average arrival rate of contacts is $\lambda$. That is to say, each node will meet with another node every $\frac{1}{\lambda}$ seconds on average. The random waypoint mobility model implies the arrival of contacts is a Poisson random process, i.e. the number of contacts a node meets within $t$ seconds is a random variable $X(t)$ and the distribution of $X(t)$ is $P(X(t)=x)=\frac{(\lambda t)^{x}}{x !} e^{-\lambda t}$, where $\lambda$ is the contact arrival rate and we assume all nodes in the network have the same contact arrival rate.

\section{B. The Model}

We consider a DTN with $N$ mobile nodes. $M$ is the maximum number of copies a message can have and $M \leq N$. At the very beginning, the source node generates a message and the source is the only carrier of that message. When the source node meets with other nodes, the source node will forward message copies to them. Other relay nodes may also forward the message and introduce more relay nodes carrying the message. For that message in concern, we define the number of relay nodes carrying that message as the "state" of the system. Obviously, the number of relay nodes carrying the message can vary from 1 to $M$. We use $S_{i}$ to denote the state of the network system in which there are $i$ relay nodes carrying the message in concern. There is another state, $S_{\text {end }}$, in the system, which represents the state that one relay node carrying the message has forwarded the message to the destination node and the message transmission is finished.

When the network system is in state $S_{i}$, it can transfer to state $S_{i+1}$, which means a relay node has forwarded a message copy to another node who does not have that message. The state $S_{i}$ can also transfer to state $S_{\text {end }}$, in case that one of the $i$ relay nodes carrying the message has met with the destination node and the message transmission is finished. We show the state transferring in Fig. 1. In this figure, the maximum number of relay nodes is $M$ and there are $M+1$ states, including the $S_{\text {end }}$ state. The parameter $a_{i}$ is the transfer rate from state $S_{i}$ to $S_{i+1}$ and $b_{i}$ is the transfer rate from state $S_{i}$ to state $S_{\text {end }}$. The random process shown in Fig. 1 is a continuous time Markov chain with absorbing state [16]. Once the transfer rates between states are known, we can have transient analysis of this Markov chain.

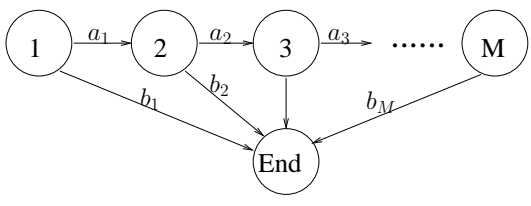

Fig. 1. The continuous time Markov model representing the message delivery in redundancy-based routing schemes.

We are particularly interested in the probability distribution of state $S_{\text {end }}, f_{\text {end }}(t)$, which is the distribution of the message delivery delay. Let $F_{i}(t)$ and $F_{\text {end }}(t)$ denote the probability that at time $t$ the system is in state $S_{i}$ and $S_{\text {end }}$, respectively. The initial state of the Markov chain is $S_{1}$; that is $F_{1}(0)=1$, $F_{i}(0)=F_{\text {end }}(0)=0$ for $i \in[2, M]$. Following Kolmogorov's equation, we have the differential equations about the state probabilities $F_{\text {end }}(t)$ and $F_{i}(t)$ for $i \in[1, M]$ shown in (1).

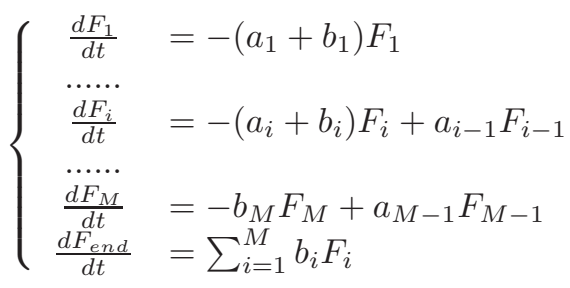

Given $F_{1}(0)=1$ and $F_{i}(0)=F_{\text {end }}(0)=0$ for $i \in[2, M]$, we can solve this system using Laplace transforms. If we define $a_{0}=1$ and $a_{M}=0$, we can derive $\bar{F}_{i}$ and $s \bar{F}_{\text {end }}$ 
as the following, in which $\bar{F}_{i}(s)$ is the Laplace transfer of $F_{i}(t), \bar{F}_{i}(s)=\mathcal{L}\left(F_{i}(t)\right)$.

$$
\left\{\begin{array}{l}
\bar{F}_{i}=\prod_{j=1}^{i} \frac{a_{j-1}}{s+a_{j}+b_{j}} \\
s \bar{F}_{\text {end }}=\sum_{i=1}^{M} b_{i} \prod_{j=1}^{i} \frac{a_{j-1}}{s+a_{j}+b_{j}}
\end{array}\right.
$$

Since $F_{\text {end }}(t)$ is the CDF of message transmission delay, we can derive the PDF of the delay as $\frac{d F_{\text {end }}(t)}{d t}=f_{\text {end }}(t)$. It follows that $\bar{f}_{\text {end }}(s)=s \bar{F}_{\text {end }}(s)-\bar{F}_{\text {end }}(0)=s \bar{F}_{\text {end }}(s)$. Considering the initial state $\bar{F}_{\text {end }}(0)=0, \bar{f}_{\text {end }}(s)$ can be written as:

$$
\bar{f}_{\text {end }}(s)=\sum_{i=1}^{M} b_{i} \prod_{j=1}^{i} \frac{a_{j-1}}{s+a_{j}+b_{j}}
$$

The inverse Laplace transform of (2) is the convolution of a series of exponential functions, as shown in (3), in which the symbol $\otimes$ represents convolution operation.

$$
f_{\text {end }}(t)=\sum_{i=1}^{M} b_{i} \bigotimes_{j=1}^{i} a_{j-1} e^{-\left(a_{j}+b_{j}\right) t}
$$

Function $f_{\text {end }}(t)$ is easy to compute because the convolution of two exponential functions is $e^{-a t} \otimes e^{-b t}=\frac{e^{-a t}-e^{-b t}}{b-a}$, when $a$ is not equal to $b$. In case of $a=b$, the convolution of $K$ exponential function is $\bigotimes_{i=1}^{K} e^{-a t}=\frac{t^{K-1}}{(K-1) !} e^{-a t}$.

\section{Using the Model to Analyze Different FORWARDING SCHEMES}

\section{A. Analysis of Direct Transmission}

In the direct transmission scheme, the source node will carry the message until it meets with the destination node. That is to say the source will not forward the message to any other node except the destination node. Because there are $(N-1)$ other nodes in the network but only one of them is the destination of the message, any contact of the source node has a $\frac{1}{N-1}$ chance to be the destination node. Under the assumption that the contact arrival is a Poisson process with arrival rate $\lambda$, the probability distribution of the delivery delay is exponential distribution.

$$
f_{\text {end }}(t)=\frac{\lambda}{N-1} e^{-\frac{\lambda t}{N-1}}
$$

We can also derive (4) from the differential equation set in (1). In this case, there are only two states in the Markov chain, state $S_{1}$ and $S_{\text {end }}$. The state transfer rate from $S_{1}$ to $S_{\text {end }}$ is $\frac{\lambda}{N-1}$. We have the following two differential equations:

$$
\left\{\begin{array}{l}
\frac{d F_{1}}{d t}=-\frac{\lambda}{N-1} F_{1} \\
\frac{d F_{e n d}}{d t}=\frac{\lambda}{N-1} F_{1}
\end{array}\right.
$$

By the Laplace transforms, we have $s \bar{F}_{\text {end }}=\frac{\lambda}{N-1} \frac{1}{s+\frac{\lambda}{N-1}}$. So the delay distribution is $f_{\text {end }}(t)=\frac{\lambda}{N-1} e^{-\frac{\lambda}{N-1} t}$.

\section{B. Analysis of Source Forward}

In source forward scheme, only the source of the message can forward copies of that message to other relay nodes. After receiving a message copy from the source, the relay node will not send that message copy to any node except the destination. Before we begin the analysis source forward routing scheme, we'd like to answer the following question: when there are $I$ nodes carrying the message, how long it takes for the first one to meet with the destination node.

For any relay node carrying the message, the average arrival rate of the destination is $\frac{1}{N-1} \lambda$. Since the contact arrival is Poisson distribution, the inter-arrival time of contact is exponential distribution $f(t)=\frac{\lambda}{N-1} e^{-\frac{\lambda}{N-1} t}$. We consider the message delivery delay when there are $I$ relay nodes carrying the message. The time needed for relay node $i$ to meet with the destination node is an exponential distributed random variable $\Phi_{i}$. The time needed for the first one of the $I$ relay nodes to encounter the destination node is $\min \left[\Phi_{1}, \ldots \Phi_{I}\right]$. If we assume $\Phi_{i}$ for $i \in[1, I]$ are IID (independent and identical distributed) random variables, we can derive the distribution of $\min \left[\Phi_{1}, \ldots \Phi_{I}\right]$ as following ${ }^{1}$ :

$$
f_{\min \left[\Phi_{1}, \ldots \Phi_{I}\right]}(t)=\frac{I \lambda}{N-1} e^{-\frac{I \lambda}{N-1} t}
$$

From (5) we see that $\min \left[\Phi_{1}, \ldots \Phi_{I}\right]$ is an exponential random variable, whose mean is $\frac{I \lambda}{N-1}$. So when there are $i$ relay nodes carrying the message copies, the transfer rate from state $S_{i}$ to state $S_{\text {end }}$ is:

$$
r\left(S_{i}, S_{\text {end }}\right)=b_{i}=\frac{i}{N-1} \lambda
$$

When there are $i$ relay nodes in the network carrying the message and the $i<M$, the state transfer rate from $S_{i}$ to $S_{\text {end }}$ is $\frac{i}{N-1} \lambda$. The transfer rate from $S_{i}$ to state $S_{i+1}$ is $\frac{N-i-1}{N-1} \lambda$, because the source node will not forward message copies to those node already having that message and the probability that the source node encounters a node who does not have the message is $\frac{N-i-1}{N-1}$, since there are $(N-i-1)$ nodes in the network can be potential relay nodes of the message.

$$
r\left(S_{i}, S_{i+1}\right)=a_{i}=\frac{N-i-1}{N-1} \lambda
$$

Replacing $a_{i}$ and $b_{i}$ in (1) with (6) and (7). We can derive the distribution of delay in source forward as shown in (8).

$$
\left\{\begin{array}{l}
f_{\text {end }}(t)=\sum_{i=1}^{M} \bigotimes_{j=1}^{i} a_{j-1} e^{-\left(a_{j}+b_{j}\right) t} \\
a_{0}=1, a_{M}=0 \\
a_{i}=\frac{N-i-1}{N-1} \lambda, \quad i \in[1, M-1] \\
b_{i}=\frac{i}{N-1} \lambda, \quad i \in[1, M]
\end{array}\right.
$$

Note $a_{i}+b_{i}=1$ for $i \in[1, M-1]$, we can write $f_{\text {end }}(t)$ as:

$$
\begin{aligned}
f_{\text {end }}(t)= & \sum_{i=1}^{M-1} b_{i}\left(\prod_{j=1}^{i} a_{j-1}\right) \frac{t^{i-1}}{(i-1) !} e^{-\lambda t} \\
& +\frac{b_{M} \prod_{j=1}^{M} a_{j-1}}{(M-2) !} t^{M-2} e^{-\lambda t} \otimes e^{-\frac{M}{N-1} \lambda t}
\end{aligned}
$$

The convolution of $t^{M-2} e^{-\lambda t}$ and $e^{-\frac{M}{N-1} \lambda t}$ is:

$$
\begin{aligned}
& t^{M-2} e^{-\lambda t} \otimes e^{-\frac{M}{N-1} \lambda t} \\
& \quad=\frac{(M-2) !\left[e^{-\frac{M}{N-1} \lambda t}-e^{-\lambda t} \sum_{i=0}^{M-2} \frac{\left(\frac{N-M-1}{N-1} \lambda t\right)^{i}}{i !}\right]}{\left(\frac{N-M-1}{N-1} \lambda\right)^{M-1}}
\end{aligned}
$$

\footnotetext{
${ }^{1}$ The distribution can be calculated like this: $P\left(\min \left[\Phi_{1}, \ldots \Phi_{I}\right] \leq x\right)=$ $1-P\left(\min \left[\Phi_{1}, \ldots \Phi_{I}\right]>x\right)=1-e^{-\frac{I \lambda}{N-1}} x \Rightarrow f_{\min \left[\Phi_{1}, \ldots \Phi_{I}\right]}(t)=$ $\frac{I \lambda}{N-1} e^{-\frac{I \lambda}{N-1} t}$
} 


\section{Analysis of Binary Forward}

Binary forward is first proposed in [3]. In this scheme, when a relay node having more them one message quota meets with another node who does not have a copy of that message, they will share the message quotas and each of them will have half of the quotas. Similarly, the transfer rate from state $S_{i}$ to state $S_{\text {end }}$ is still the following:

$$
r\left(S_{i}, S_{\text {end }}\right)=b_{i}=\frac{i}{N-1} \lambda
$$

In order to derive the transfer rate from state $S_{i}$ to $S_{i+1}$, we need to consider the following question: When there are $i$ nodes in the network carrying the message copies (there are $i$ relays for a message), how many of them have the ability to forward message copy to other nodes. We approximate this problem using the results of integer partition [17], which is a well studied problem in combinatorics. Let $P(D \mid k)$ denote the number of different partitions when an integer $D$ is partitioned into $k$ parts. We have the following recursive function about $P(D \mid k)$ [17]:

$$
P(D \mid k)=\sum_{i=1}^{k} P(D-k \mid i)
$$

$P(D \mid k)$ can be recursively computed by noticing that $P(D \mid k)=1$ for $D=k$ and $P(D \mid k)=0$ for $D<k$.

Suppose there are $P(D \mid k)$ different ways to partition $D$ into $k$ parts, $D=x_{1}+x_{2}+\ldots+x_{k}, x_{1} \geq x_{2} \geq \ldots x_{k} \geq 1$. We want to know how many ways we have when we partition $D$ into $k$ parts and $s$ out of the $k$ parts are larger than 1 . That is to say $D-k=\left(x_{1}-1\right)+\left(x_{2}-1\right)+\ldots+\left(x_{k}-1\right)=x_{1}^{\prime}+x_{2}^{\prime}+\ldots+x_{s}^{\prime}$, $x_{1}^{\prime} \geq x_{2}^{\prime} \geq \ldots x_{s}^{\prime} \geq 1$. To partition $(D-k)$ into $s$ parts, there are $P(D-k \mid s)$ different ways. Therefore, when we partition $D$ into $k$ parts, on average there are $\frac{\sum_{s=1}^{k} s P(D-k \mid s)}{P(D \mid k)}$ parts larger than 1. Back to our problem in binary forward, when there are $k$ relay nodes carrying message copies, we can use the $P^{\prime}(M \mid k)$ in (9) to approximate the average number of nodes who can forward their message copy to other relay nodes who do not have that message.

$$
P^{\prime}(M \mid k)=\frac{\sum_{s=1}^{k} s P(M-k \mid s)}{P(M \mid k)}
$$

The transfer rate from state $S_{i}$ to state $S_{i+1}$ can be written as the following:

$$
r\left(S_{i}, S_{i+1}\right)=a_{i}=P^{\prime}(M \mid i) \frac{N-i-1}{N-1} \lambda
$$

Now we have all the transfer rates. Using the same technique in Section IV-B we can get the distribution of the message delay in case of binary forward $\bar{f}_{\text {end }}(t)$ as shown in (11).

$$
\left\{\begin{array}{l}
f_{\text {end }}(t)=\sum_{i=1}^{M} b_{i} \bigotimes_{j=1}^{i} a_{j-1} e^{-\left(a_{j}+b_{j}\right) t} \\
a_{0}=1, a_{M}=0 \\
a_{i}=P^{\prime}(M \mid i) \frac{N-i-1}{N-1} \lambda, \quad i \in[1, M-1] \\
b_{i}=\frac{i}{N-1} \lambda, \quad i \in[1, M]
\end{array}\right.
$$

\section{EXPERIMENT Evaluation AND Discussion}

\section{A. Simulation Setup}

We use the random way point mobility model [18] in our simulation experiments. Existing work has shown that the contact inter-arrival time in random way point mobility model is exponential distribution [19]. We have 100 nodes move in a disc with radius set to 1 unit and there is no node failure. All nodes have the same moving speed and the same communication range. The initial positions of nodes are randomly chosen in the disc. There is no thinking time after nodes arrive at the way points. We set 10000 different random seeds to run the simulation program so we have 10000 simulation instances. We use the message delivery delay in those results to plot the probability distribution of the delay. For convenience of reading, we normalize the Y-axis to $0 \sim 1$ for all the following plots.

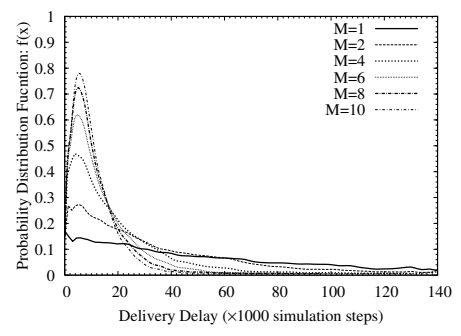

(a) simulation result

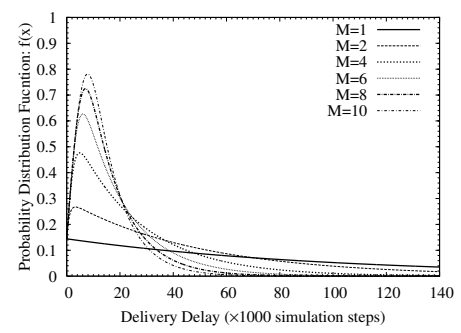

(b) analytic result
Fig. 2. Probability distribution of message delivery delay in source forward.

\section{B. Simulations of Source Forward}

We set the number of maximum copies of a message, $M$, to different values and run the simulation for each value for 10000 times. The simulation results are plotted in Fig. 2(a). We also plot the probability distributions derived from the analytic result in Fig. 2(b). From the plots in Fig. 2 we can see that the probability distributions derived from our analytical model have almost the same shapes as the simulation results. When the parameter $M$ is 1 , the message forwarding is equivalent to the direct transmission scheme and the message delivery delay is an exponentially distributed function. When multiple copies of a message are generated into the network, the message delivery delay function has a hill-like shape.

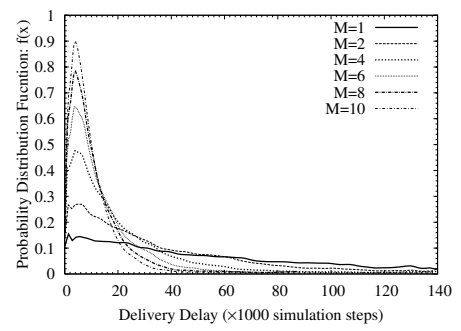

(a) simulation result

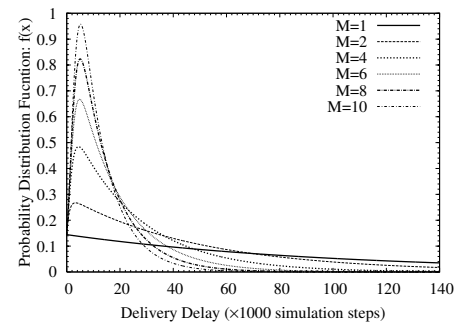

(b) analytic result
Fig. 3. Probability distribution of message delivery delay in binary forward.

\section{Simulations of Binary Forward}

Fig. 3(a) shows the simulation results of the delay probability distribution when binary forward is used. The analytical results are shown in Fig. 3(b) as well. From the plots in Fig. 3 we can see that the probability distributions in case 


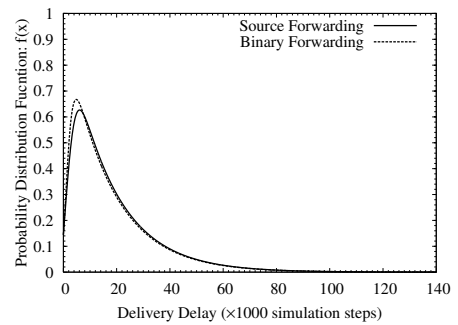

(a) $\mathrm{M}=6$

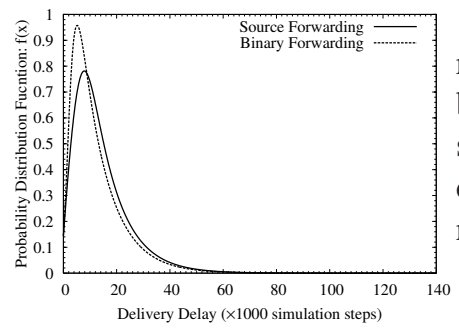

(b) $\mathrm{M}=10$
Fig. 4. Compare between source and binary forward schemes when setting the maximum number of redundant message copies, $M$, to different values.

of binary forward still have hill-like shapes when multiple copies are used, which well matches the simulation results. But the curves of binary forward scheme increase more rapidly than the distribution function of source forward scheme before reaching their highest points.

\section{Discussions}

As we have shown in Fig. 2 and Fig. 3, the modeling results match pretty well with the simulation experiment results. We plot the delivery delay distributions of source forward and binary forward schemes together in Fig. 4 according to the analytic results shown in (8) and (11), where the maximum number of message copies is set to 6 and 10, respectively.

1) Characteristics of the Delay Distribution Functions: From the plot in Fig. 4 we can see that the delivery delay distributions are quite different from the exponential distribution, although the contact arrival is exponential distribution, which is a monotonically decreasing function. For source and binary forward, the delay distribution functions start from a low value and increase rapidly until reaching their maximum values. After that, the distribution functions decrease similarly to exponential functions.

From the plot in Fig. 4 we can see that how the source distributes the message copies has significant impact on message delivery, especially when the maximum number of message copies, $M$, is set to a relatively high value. In order to achieve fast message delivery, we should carefully design how to distribute the message copies. In those networks where nodes are identical, binary forward is optimal. But for networks where nodes have different attributions, such as moving velocity and buffer capacity, we need more sophisticated schemes. We leave this issue as the future work of this paper.

2) Applications of the Derived Delay Distribution: The insight of the delay distribution function can help us in the design of redundancy-based routing in DTNs. In realistic DTNs, the mobile nodes have finite buffers. There needs some message drop strategies in case of buffer overflow. One commonly used strategy is dropping those messages who were generated long before. This is similar to set the TTL field in the IP header in today's Internet. The delay distribution function can provide us with a guideline on how to set the lifetime of a message. For example, if the delay distribution function shows that $90 \%$ of delays are less than $T$, we can say that most of the messages are likely to be successfully delivered when we set the message lifetime to $T$.
The analytical results can also be used in determining the replicate factor $M$. Intuitively, if we want more messages to be delivered within certain time, more message redundancy should be used. Given the delay requirement and the percentage of messages should be delivered, we can derive the replicate factor $M$ using our analytical results.

\section{CONCLUSION}

In this paper, we have presented a continuous time Markov random process model for modeling the message transmission in redundancy-based routing schemes for delay tolerant networks. With this model, we derived the probability distribution functions of a class of redundancy-based DTN routing schemes. We use simulation experiments to verify our model. The results show that our analytical model can precisely capture the characteristics of message delivery in redundancy-based message routing schemes.

\section{REFERENCES}

[1] K. Fall, "A delay-tolerant network architecture for challenged Internets," in SIGCOMM '03. New York, NY, USA: ACM Press, 2003, pp. 27-34.

[2] P. Juang, H. Oki, Y. Wang, M. Martonosi, L. S. Peh, and D. Rubenstein, "Energy-efficient computing for wildlife tracking: design tradeoffs and early experiences with zebranet," SIGOPS Oper. Syst. Rev., vol. 36, no. 5, pp. 96-107, 2002.

[3] T. Spyropoulos, K. Psounis, and C. Raghavendra, "Spray and wait: an efficient routing scheme for intermittently connected mobile networks," in WDTN '05: SIGCOMM'05 DTN workshop, 2005, pp. 252-259.

[4] Z. Zhang, "Intermittently connected mobile ad hoc networks and delay tolerant networks: Overview and challenges," IEEE Communication Survey and Tutorial, first quarter, 2006.

[5] S. Jain, K. Fall, and R. Patra, "Routing in a delay tolerant network," in SIGCOMM' 04, Aug. 2004.

[6] S. Merugu, M. Ammar, and E. Zegura, "Routing in space and time in networks with predictable mobility," Georgia Institute of Technology, Technical Report GIT-CC-04-7, 2004.

[7] T. Spyropoulos, K. Psounis, and C. Raghavendra, "Single-copy routing in intermittently connected mobile networks," in Secon'05, Oct. 2004.

[8] A. Lindgren, A. Doria, and O. Schelen, "Probabilistic routing in intermittently connected networks," SIGMOBILE Mob. Comput. Commun. Rev., vol. 7, no. 3, pp. 19-20, 2003.

[9] K. Tan, Q. Zhang, and W. Zhu, "Shortest path routing in partially connected ad hoc networks," in Globalcom, 2003.

[10] J. Leguay, T. Friedman, and V. Conan, "DTN routing in a mobility pattern space,' in WDTN '05: SIGCOMM'05 DTN workshop, 2005, pp. $276-283$.

[11] D. B. Amin Vahdat, "Epidemic routing for partially connected ad hoc networks," Duke University, Technical Report CS-200006, Apr. 2000.

[12] T. Spyropoulos, K. Psounis, and C. S. Raghavendra, "Multi-copy routing in intermittently connected mobile networks," USC," Technical Report, 2004.

[13] Y. Wang, S. Jain, M. Martonosi, and K. Fall, "Erasure-coding based routing for opportunistic networks," in WDTN '05: SIGCOMM'05 DTN workshop, 2005, pp. 229-236.

[14] Y. Liao, K. Tan, Z. Zhang, and L. Gao, "Estimation based erasurecoding routing in delay tolerant networks," in $I W C M C$ ' 06: International Wireless Communications and Mobile Computing Conference, Delay Tolerant Mobile Networks Symposium, Vancouver, Canada, July 2006.

[15] P. T. Eugster, R. Guerraoui, A.-M. Kermarrec, and L. Massoulieacute;, "Epidemic information dissemination in distributed systems," Computer, vol. 37, no. 5, pp. 60-67, 2004.

[16] K. S. Trivedi, Probability and statistics with reliability, queuing and computer science applications. Chichester, UK, UK: John Wiley and Sons Ltd., 2002.

[17] K. P. Bogart, Introductory Combinatorics. Orlando, FL, USA: Academic Press, Inc., 2000.

[18] "Random waypoint model." [Online]. Available: http://www.netlab.hut. fi/u/esa/java/rwp/rwp-model.shtml

[19] N. Bansal and Z. Liu, "Capacity, delay and mobility in wireless ad-hoc networks," in INFOCOM' 03, 2003. 